\title{
Terlipressin infusion induces Tako-Tsubo syndrome in a cirrhotic man with hepato-renal syndrome
}

\author{
Antonio Di Micoli • Daniela Buccione · Daniela Degli Esposti • Valentina Santi • \\ Luciana Bastagli · Claudio Borghi · Mauro Bernardi · Franco Trevisani
}

Received: 8 November 2010/Accepted: 24 January 2011/Published online: 15 February 2011

(c) SIMI 2011

\section{Introduction and case presentation}

Dr. Di Micoli, Dr. Buccione, Prof. Trevisani: TakoTsubo cardiomyopathy (TTC), also known as transient left ventricular apical ballooning syndrome, is a clinical entity characterized by (1) reversible left ventricular apical wall motion abnormalities, (2) typical electrocardiographic changes and (3) relatively minor elevation of troponine, creatinine-kinase $(\mathrm{CK})$ and $\mathrm{CK}-\mathrm{MB}$ that mimics an acute myocardial infarction (AMI) without any acute obstructive coronary disease [1]. Typically, the left ventricular imbalance almost always recovers in a period of days to weeks, so that the management and prognosis of this condition are clearly different from those of AMI [2]. TTC is generally observed in post-menopausal women without prior history of heart disease or clear risk factors for coronary artery disease, who have often experienced recent emotional or physical stress, non-cardiac surgery or extracardiac diseases [1]. Recently, TTC has also been

A. Di Micoli · D. Buccione - V. Santi - L. Bastagli .

M. Bernardi · F. Trevisani

Dipartimento di Medicina Clinica,

Alma Mater Studiorum-Università degli

Studi di Bologna, Bologna, Italy

A. Di Micoli $(\bowtie)$

Dipartimento di Medicina Clinica, U.O. Semeiotica Medica, Azienda Ospedaliero-Universitaria Policlinico

Sant'Orsola-Malpighi, via Albertoni, 15,

40138 Bologna, Italy

e-mail: antoniodimicoli@libero.it

D. Degli Esposti · C. Borghi

Dipartimento di Medicina Interna, dell'Invecchiamento e Malattie Nefrologiche, Alma Mater Studiorum-Università degli

Studi di Bologna, Bologna, Italy described in critically ill patients without prior heart disease admitted to a medical intensive care unit for severe non-cardiac diseases, such as sepsis, acute respiratory failure, systemic inflammatory response syndrome, anaphylaxis and trauma injuries [3, 4].

Herein, we report a case of TTC occurring in a cirrhotic man, waiting for liver transplantation (LT), probably favored by the intravenous infusion of terlipressin, a synthetic analog of vasopressin (AVP), for the treatment of hepato-renal syndrome (HRS).

A 67-year-old Caucasian man with hepatitis B virusrelated cirrhosis and ascites, waiting for LT, was admitted to our unit because of the onset of hepatic encephalopathy, and the worsening of renal sodium retention. As a candidate for LT, he had undergone clinical, laboratory and instrumental tests aimed at excluding extrahepatic diseases precluding surgery. Myocardial single photon emission computed tomography had excluded ischemic damage, and trans-thoracic echocardiography had ruled out dyskinesia of ventricular segments and documented a normal (65\%) left ventricular ejection fraction (LVEF).

On admission, the patient presented with peripheral edema, ascites and grade III hepatic encephalopathy. Daily diuresis was around $200 \mathrm{~mL}$. The ongoing therapy included oral diuretics (furosemide $25 \mathrm{mg}$ b.i.d. and spironolactone $100 \mathrm{mg}$ b.i.d.), lamivudine and tenofovir for HBV infection control, norfloxacine ( $400 \mathrm{mg} /$ day) for secondary prophylaxis of the spontaneous peritoneal peritonitis, lactulose, and periodic albumin infusion. The serum creatinine was $2.2 \mathrm{mg} / \mathrm{dL}$ and blood urea nitrogen $0.9 \mathrm{~g} / \mathrm{dL}$, while serum electrolyte concentrations were normal (sodium $138 \mathrm{mEq} / \mathrm{L}$, potassium $3.5 \mathrm{mEq} / \mathrm{L}$, calcium $8.9 \mathrm{mg} / \mathrm{dL}$ and magnesium $2.6 \mathrm{mEq} / \mathrm{L}$ ). The hemoglobin level was $10.4 \mathrm{~g} / \mathrm{dL}$, platelet count $134.000 / \mu \mathrm{L}$, leukocytes $8.0 \times 10^{3} / \mathrm{mmc}$, serum bilirubin $18.8 \mathrm{mg} / \mathrm{dL}$, albumin $3.4 \mathrm{~g} / \mathrm{dL}$, INR 2.11 . 


\section{Preliminary diagnosis}

Dr. Di Micoli, Dr. Buccione, Dr. Santi: Bacterial and fungal infections, pulmonary diseases or embolism and urinary obstructive disease were ruled out by medical history, physical examination, chest X-ray study, pulmonary scintigraphy, abdominal ultrasonography, and blood and urine tests. The electrocardiogram (EKG) was normal (sinus rhythm, heart rate $80 \mathrm{bpm}$, absence of ST abnormalities) with the exception of a pre-existing right bundle branch block. Soon after admission, diuretics were withdrawn and plasma volume expansion was started (saline $1,500 \mathrm{~mL}$ ) without any favorable effect on serum creatinine. The diagnosis of type 2 HRS was postulated, and, according to the international guidelines available at that time [5], terlipressin administration was started at the dose of $3 \mathrm{mg} /$ day (continuous infusion) plus i.v. albumin $(1 \mathrm{~g} / \mathrm{kg}$ of body weight). Renal function improved (creatinine $1.5 \mathrm{mg} / \mathrm{dL}$ ) on the third day of hospitalization, but the patient complained of tachycardia (110 bpm), chest pain, and dyspnea, and the QT interval, corrected by the heart rate [6], lengthened from 454 to $490 \mathrm{~ms}$. A minimal elevation $(0.04 \mathrm{ng} / \mathrm{mL}$, normal range $\leq 0.03)$ of serum troponine I (cTn-I) was found, without concurrent elevation of CPK and CPK-MB, but the subsequent values, checked after 6 and $12 \mathrm{~h}$, were normal.

\section{Further investigation and differential diagnosis}

\section{Dr. Buccione, Dr. Santi, Dr. Degli Esposti: Serial EKGs} did not show features compatible with acute coronary syndrome. Conversely, a trans-thoracic echocardiography disclosed akinesia of mid-apical segments with systolic ballooning of the left ventricular apex, hyperkinetic motion of the basal segments, and marked reduction of LVEF (30\%) (Fig. 1), suggesting the classic form of TTC [7]. The infusion of terlipressin was immediately stopped, and oral metoprolol (50 mg b.i.d.) was given. The patient was temporarily removed from the LT list. Cardiac magnetic resonance (CMR) was performed, but was not diagnostic because it was impossible to synchronize the very low EKG signal with CMR; indeed, the EKG signal was affected by both the cardiac impairment and the anasarca. The impairment of renal function (creatinine $2.2 \mathrm{mg} / \mathrm{dL}$ ) that followed terlipressin withdrawal prevented the performance of a planned coronary aortography.

\section{Clinical evolution and follow up}

Dr. Di Micoli, Dr. Degli Esposti, Dr. Bastagli: After terlipressin withdrawal, chest pain and dyspnea quickly resolved, and the QTc interval length shortened to the initial value at the EKG recorded $5 \mathrm{~h}$ later. A second transthoracic echocardiography, performed 1 day later, did not show improvement, whereas the third assessment, made 4 days later, demonstrated the resolution of the mid-apical segments akinesia and normalization of LVEF (70\%), testifying to the short-life of these abnormalities. Over the following days, the clinical condition and renal function of the patient improved, and diuretic therapy was restarted. The patient was then re-listed for LT. Unfortunately, 1 week later he developed severe sepsis. His clinical condition quickly worsened, and he died on the 20th day of hospitalization.

\section{Discussion}

Dr. Di Micoli, Prof. Borghi, Prof. Bernardi, Prof. Trevisani: To the best of our knowledge, this is a unique case of a TTC occurring in a patient with hepatic cirrhosis. This syndrome, also called stress-induced cardiomyopathy, was first described in Japanese women, [8] and is characterized by reversible left ventricular and apical wall motion
Fig. 1 Two dimensional echo images of the left ventricle in apical four-chamber view. a At end-diastole, the left ventricle shows substantially normal dimensions and wall thickening with minimally dilated apical segments. b At end-systole, the left ventricular chamber shows the typical apical ballooning (arrow) due to the akinesia/ hypokinesia of apical and midsegments. $L V$ left ventricle, $R V$ right ventricle, $L A$ left atrium, $R A$ right atrium
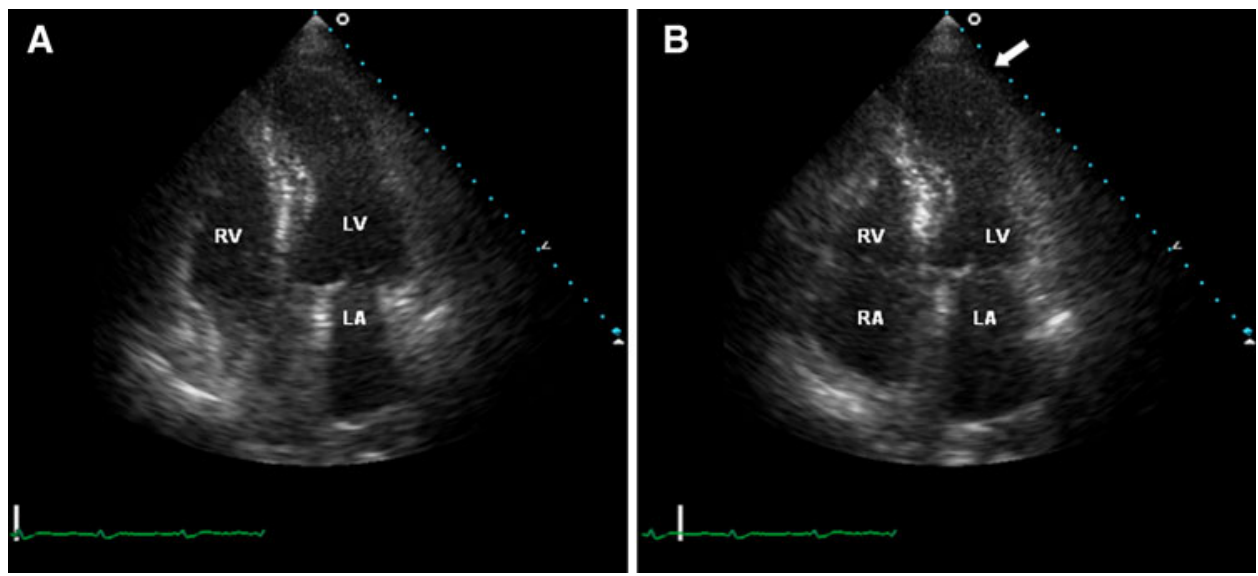
abnormalities, ischemic type chest pain, EKG changes (including ST-segment elevation, especially in the precordial leads, abnormal Q waves, prolongation of QT interval, and rapid evolution of symmetric negative T-waves in most leads [9]) and a slight elevation of myocardial enzymes, without angiographic evidence of epicardial coronary stenosis. In our patient, the diagnosis of TTC was supported by the lack of EKG changes compatible with acute ischemic damage, the minimal and transient increase of serum CTn-I and, above all, the typical echocardiographic features [7] that fully disappeared in a few days, suggesting their functional nature.

The precise causes of TTC are still undefined, and several pathophysiological mechanisms have been postulated. They include epicardial coronary spasm, excess sympathetic stimulation, microvascular dysfunction, and dynamic left ventricular outflow obstruction [1].Considering the frequent association between TTC and emotional or physical stress, this syndrome has been claimed as a "catecholamine-induced" myocardial injury, and sympathetic hyperactivity at the cardiac apex and high plasma catecholamines have been reported in several cases $[9,10]$. Furthermore, in a rat model of TTC caused by immobilization stress, the activation of $\alpha$ - and $\beta$-adrenoreceptors is the main trigger of the transient left ventricular apical ballooning [11]. Lastly, recent studies demonstrate that patients with TTC exhibit impaired myocardial perfusion, coronary microvascular dysfunction [9] and metabolic abnormalities in the left ventricular apex similar to those observed in post-ischemic myocardial stunning [12].

The peculiarities of our case are: (1) the unusual gender and clinical background of the patient, TTC being much more frequent in women and never before described in advanced hepatic cirrhosis; (2) the absence of a previous emotionally stressful event, although the cardiac dysfunction ensued on top of a severe non-cardiac illness, such as type 2 HRS; (3) the onset of TCC during the infusion of terlipressin, a potent non-catecholaminergic vasoconstrictor, and its rapid improvement after withdrawal of this drug.

AVP and its pro-drug terlipressin have a marked and multiorgan vasoconstrictor effect, mainly through the $\mathrm{V}_{1 \mathrm{a}}$ receptors, but also by the ATP-sensitive $\mathrm{K}^{+}$channels [13]. The AVP effect on the coronary vascular bed is intensified by an inhibition of the endothelial production of nitric oxide [14]. Moreover, pharmacological doses of AVP reduce cardiac output independently of the increase in coronary vascular resistance. [15]. Lastly, in isolated rat heart, when coronary perfusion is maintained constant, low concentrations of AVP enhance the myocardial contractility, whereas an opposite effect is observed at high AVP concentrations $(500 \mathrm{pg} / \mathrm{mL})$ [16]. In clinical practice, terlipressin is preferred to AVP because of less pronounced effects on the coronary circulation. In fact, a coronary vasoconstriction is appreciable only at supra-therapeutic concentrations of $30 \mathrm{nM}$ in isolated perfused rabbit heart [17]. Nonetheless, therapeutic doses of terlipressin have been reported to provoke cardiac ischemic effects in patients with intraoperative hypotension [18] and in cirrhotic patients with HRS [19].

It can be suspected that, in our patient, TTC was precipitated by the combination of very high plasma levels of catecholamines, a typical feature of type 2 HRS [20], and the terlipressin infusion, which contributes to induce a marked vasoconstriction in the coronary bed via the stimulation of non-adrenergic receptors. The resulting ischemia could have produced the cardiac dysfunction typical of TTC in a basically non-ischemic heart. Moreover, as terlipressin has a renal clearance, a depressed excretion could have increased its plasma concentration in our patient, favoring the development of its adverse effects.

Our report expands the clinical settings in which TTC may be observed, showing that it can affect cirrhotic patients with predisposing clinical factors, such as acute stressful clinical events, and the infusion of non-catecholaminergic vasoconstrictor agents.

\section{Conflict of interest None.}

\section{References}

1. Hansen PR (2007) Takotsubo cardiomyopathy: an under-recognized myocardial syndrome. Eur J Intern Med 18:561-565

2. Park SM, Prasad A, Rihal C, Bell MR, Oh JK (2009) Left ventricular systolic and diastolic function in patients with apical ballooning syndrome compared with patients with acute anterior ST-segment elevation myocardial infarction: a functional paradox. Mayo Clin Proc 84:514-521

3. Ruiz Bailén M (2002) Reversible myocardial dysfunction in critically ill noncardiac patients: a review. Crit Care Med 30:1280-1290

4. Zanobetti M, Vicidomini S, Conti A, Innocenti F, Pini R (2010) An atypical case of inverted Tako-Tsubo syndrome: case report and review of the literature. Intern Emerg Med 5:215-219

5. Salerno F, Gerbes A, Ginès P, Wong F, Arroyo V (2007) Diagnosis, prevention and treatment of hepatorenal syndrome in cirrhosis. Gut 56:1310-1318

6. Zambruni A, Di Micoli A, Lubisco A, Domenicali M, Trevisani F, Bernardi M (2007) QT interval correction in patients with cirrhosis. J Cardiovasc Electrophysiol 18:77-82

7. Desmet WJ, Adriaenssens BF, Dens JA (2003) Apical ballooning of the left ventricle: first series in white patients. Heart 89:10271031

8. Dote K, Sato H, Tateishi H, Uchida T, Ishihara M (1991) Myocardial stunning due to simultaneous multivessel coronary spasms: a review of 5 cases. J Cardiol 21:203-214

9. Bybee KA, Kara T, Prasad A, Lerman A, Barsness GW, Wright RS, Rihal CS (2004) Systematic review: transient left ventricular apical ballooning: a syndrome that mimics ST-segment elevation myocardial infarction. Ann Intern Med 41:858-865

10. Wittstein IS, Thiemann DR, Lima JA, Baughman KL, Schulman SP, Gerstenblith G, Wu KC, Rade JJ, Bivalacqua TJ, Champion 
HC (2005) Neurohumoral features of myocardial stunning due to sudden emotional stress. N Engl J Med 352:539-548

11. Ueyama T (2004) Emotional stress-induced Tako-tsubo cardiomyopathy: animal model and molecular mechanism. Ann N Y Acad Sci 1018:437-444

12. Kurisu S, Inoue I, Kawagoe T (2003) Myocardial perfusion and fatty acid metabolism in patients with tako-tsubo-like left ventricular dysfunction. J Am Coll Cardiol 41:743-748

13. Berecek KH, Barron KW, Webb RL, Brody MJ (1982) Vasopressin-central nervous system interactions in the development of DOCA hypertension. Hypertension 4:131-137

14. García-Villalón AL, Garcia JL, Fernández N, Monge L, Gómez B, Diéguez G (1996) Regional differences in the arterial response to vasopressin: role of endothelial nitric oxide. $\mathrm{Br} \mathrm{J}$ Pharmacol 118:1848-1854

15. Maturi MF, Martin SE, Markle D, Maxwell M, Burruss CR, Speir E, Greene R, Ro YM, Vitale D, Green MV (1991) Coronary vasoconstriction induced by vasopressin. Production of myocardial ischemia in dogs by constriction of non diseased small vessels. Circulation 83:2111-2121
16. Walker BR, Childs ME, Adams EM (1988) Direct cardiac effects of vasopressin: role of V1- and V2-vasopressinergic receptors. Am J Physiol 255:261-265

17. Ouattara A, Landi M, Le Manach Y, Lecomte P, Leguen M, Boccara G, Coriat P, Riou B (2005) Comparative cardiac effects of terlipressin, vasopressin, and norepinephrine on an isolated perfused rabbit heart. Anesthesiology 102:85-92

18. Medel J, Boccara G, Van de Steen E, Bertrand M, Godet G, Coriat P (2001) Terlipressin for treating intraoperative hypotension: can it unmask myocardial ischemia? Anesth Analg 93:53-55

19. Sanyal AJ, Boyer T, Garcia-Tsao G, Regenstein F, Rossaro L, Appenrodt B, Blei A, Gülberg V, Sigal S, Teuber P (2008) Terlipressin Study Group. A randomized, prospective, doubleblind, placebo-controlled trial of terlipressin for type 1 hepatorenal syndrome. Gastroenterology 134:1360-1368

20. Krag A, Borup T, Møller S, Bendtsen F (2008) Efficacy and safety of terlipressin in cirrhotic patients with variceal bleeding or hepatorenal syndrome. Adv Ther 25:1105-1140 